\title{
LA ADMINISTRACIÓN EDUCATIVA Y SU FUNDAMENTACIÓN EPISTEMOLÓGICA
}

Flora Eugenia Salas Madriz

\author{
Recibido 15-IV-2002 • Aceptado 14-V-2002
}

\begin{abstract}
Resumen: En este artículo se establecen algunos criterios epistemológicos, desde la perspectiva de Mario Bunge y de Thomas Kuhn, para esclarecer la naturaleza disciplinar de la Administración Educativa. Estos criterios son básicos y, por ello, sólo se prentende facilitar la comprensión de los principales componentes de la Administración Educativa, en tanto disciplina adscrita a las Ciencias de la Educación, que faciliten la comprensión de su núcleo teórico-conceptual y de su praxis.
\end{abstract}

Palabras clave: Administración Educativa, Epistemología.

\section{Introducción}

Como parte de una preocupación general en la Escuela de Administración Educativa de la Facultad de Educación de la Universidad de Costa Rica, se detectó la necesidad de hacer precisiones metateóricas, orientadas a esclarecer aspectos epistemológicos centrales en el quehacer teórico y práctico de la disciplina, con el fin de revisar y atender las demandas que conlleva la nueva realidad educativa.

Además, se espera con este artículo atender la necesidad sentida por parte de los estudiantes de la carrera, en cuanto a establecer criterios para orientarse dentro de la vasta y compleja red de disciplinas que entran en juego en el estudio del fenómeno educativo. Conscientes de lo complejo de esta tarea, ofrecemos a continuación algunos aspectos epistemológicos, sin pretender por ello ni agotar el tema, ni abarcar todos los elementos que le subyacen. Se espera únicamente ofrecer criterios básicos para facilitar la comprensión de los principales componentes de la Administración Educativa en tanto disciplina adscrita a las Ciencias de la Educación, que faciliten la comprensión de su núcleo teóricoconceptual y de su praxis, a quienes se forman en la disciplina. 


\section{Antecedentes}

Desde que la educación se constituyó en el siglo XIX en un proyecto social, económico, político y cultural, como resultado de los procesos desencadenados en estos ámbitos por la Revolución Industrial y la caída de los gobiernos monárquicos, se convirtió en objeto de estudio filosófico y, posteriormente, de distintas disciplinas como la Sociología, la Psicología, y las Ciencias Políticas, entre otras. Conforme la educación fue ganando importancia dentro del nuevo orden mundial en el siglo XX, atrajo la conformación de una comunidad de investigadores, dedicados a dar cuenta de la compleja trama de elementos que entran en juego en el proceso enseñanza-aprendizaje, no sólo a nivel pedagógico, sino también administrativo. Por esta razón, la Administración, disciplina aún joven, con esasos cien años de desarrollo y evolución, fue ofreciendo aspectos epistemológicos, teóricos, metodológicos y técnicos, de importancia fundamental para el desarrollo de una teoría capaz de dar cuenta de la administración de las organizaciones educativas.

El principal logro del siglo XIX, cuya característica fue la búsqueda de una perspectiva científica de la realidad, fue la configuración de teorías en el campo de las ciencias sociales; primero, desde el positivismo $\mathrm{y}$, posteriormente, desde otras posiciones epistemológicas, ofreciendo en el siglo XX un amplio espectro de propuestas que hoy constituyen la base del extraordinario progreso científico en estas disciplinas. En el siglo XX ocurre, además, en el campo específico de la Administración una importante revolución epistemológica a partir de la puesta en escena del pensamiento sociológico alternativo al positivista de base comtiana. Precisamente, en esta línea, Max Weber propondrá no sólo una nueva epistemología para las ciencias sociales, sino que hará una distinción fundamental para la teoría administrativa: elaborará la argumentación para la diferenciación entre el objeto de estudio de la Sociología (la sociedad), y el de la Teoría de la Organización; es decir, las organizaciones (Cfr.: Chiavenato, 1995).

En el plano ontológico, la distinción entre el objeto de estudio de la Sociología y el de la Teoría de la Organización, implicó la autonomía disciplinar de esta última. Así, las organizaciones podían ser estudiadas en su especificidad, a fin de comprender su configuración interna, tanto como sus vínculos con el entorno social al que pertenecen, ofreciendo una perspectiva analítica, descriptiva y crítica de las mismas. El pensamiento administrativo se nutrirá fundamentalmente de esta teoría para poder responder a los problemas que plantean las organizaciones como entidades públicas o privadas, a nivel de productividad, empleo de recursos, función social y estructura interna. Así, el objeto de estudio de la teoría administrativa en general, es la gestión de las organizaciones; esto es, la búsqueda del logro (eficacia) de sus fines específicos con el uso más racional de los recursos (eficiencia); donde, tales fines se encuentran determinados por la naturaleza y características particulares de la organización (Cfr.: Chiavenato, 1995).

\section{El objeto de estudio de la Administración Educativa}

La organización educativa es un tipo específico de organización, cuya especificidad estriba en las características, estructura y funciones que le corresponda, según el nivel educativo de que trate; es decir, dependiendo de si se encarga de impartir educación en preescolar, primaria, secundaria, áreas técnicas, nivel parauniversitario o universitario, o sistemas no formales.

Con base en lo expuesto hasta aquí, podemos señalar que la organización educativa constituye el objeto de estudio de la Administración Educativa, y, como lo muestra el esquema No.1, la teoría de esta 
disciplina se construye a partir de la confluencia de tres ejes disciplinares fundamentales: las Ciencias de la Educación, la teoría administrativa general, y otras disciplinas, como la Economía, la Psicología, la Sociología, las Ciencias Políticas y el Derecho, entre otras.

\section{Esquema No. 1}

Objeto de estudio de la Administración Educativa y ejes teóricos fundamentales

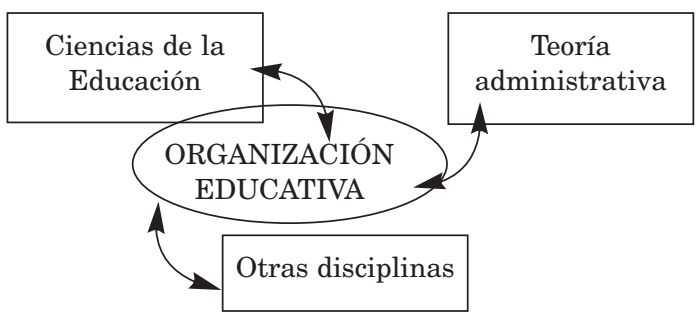

\section{La Administración Educativa como disciplina científica}

Mario Bunge (Gómez, 1996), uno de los epistemólogos latinoamericanos más importantes, propone una serie de indicadores para evaluar si una disciplina ha alcanzado status científico. Con el propósito de delimitar a la Administración Educativa como disciplina científica, a continuación se procederá a encontrar estos indicadores en los niveles teórico, metodológico y práctico.

\section{a. Existe una comunidad de investigadores en la que se da flujo de información constante e inteligente sobre los objetos de estudio}

Es un hecho que existe una comunidad de investigadores en el campo de la Administración Educativa, no sólo a nivel nacional, sino también internacional. Además, en Costa Rica existe una profesión específica a nivel universitario de bachillerato, licenciatura y de posgrado en Administración Educativa, en universidades públicas y privadas, y el nivel de doctorado en Europa y los Estados Unidos.

El hecho de que exista una estructura universitaria completa en el campo de la Administración Educativa, supone que existe, a su vez, un paradigma o matriz disciplinar (Kuhn, 1975). Así, los paradigmas son “... lo que los miembros de una comunidad científica comparten, y, recíprocamente, una comunidad científica consiste en hombres que comparten un paradigma" (Kuhn, 1975: 271). En este sentido, la vasta comunidad de docentes, estudiantes e investigadores en el campo de la Administración Educativa a nivel universitario, garantizan la consolidación y desarrollo de la misma, en función de la constante revisión y ampliación de la teoría, metodologías y aplicaciones de la disciplina que, a su vez, responden a una demanda social derivada de la necesidad de dar cuenta del fenómeno de la administración de las organizaciones educativas en todos sus niveles.

\section{b. El sistema social aprecia y valora el trabajo científico que en ella se realiza, al punto que facilita los medios necesarios para que ésto ocurra}

El Ministerio de Educación Pública, además de las universidades públicas y privadas, suponen la importancia social del trabajo científico y práctico en el campo de la Administración Educativa. Además, el Servicio Civil tiene tipificado este tipo de puesto, así como los requerimientos académicos y personales necesarios para el desempeño de puestos en el campo de la Administración Educativa.

De igual modo y como se expuso en el inciso anterior, existe una demanda social claramente definida respecto a la necesidad de resolver problemas, atender y mejorar la administración de las organizaciones educativas en todos sus niveles. Es un hecho que las nuevas necesidades del sistema económico, político y social de la era posmoderna, 
cuya base es el conocimiento, han tenido como consecuencia un incremento de la demanda por educación y, con ello, de nuevos esquemas de eficiencia, eficacia y calidad en la gestión de las organizaciones educativas.

\section{c. Los objetos que estudia son entes reales}

Como se señaló anteriormente, la Administración Educativa tiene por objeto de estudio la organización educativa, en sus distintos niveles: primario, secundario, terciario y no formal. Cada nivel educativo posee características y requerimientos diferentes, en función de la edad y necesidades de las poblaciones que atiende. Cada nivel educativo exige a la organización educativa procesos de administración distintos, en el tanto sus responsabilidades, objetivos $\mathrm{y}$ metas son diversos y obedecen a las características propias de cada etapa de formación. El grado de autonomía del aprendiz, así como los contenidos del aprendizaje varían desde la infancia hasta la adultez, en el entendido de que cada etapa del proceso educativo articula un andamiaje que hace posible que las personas accedan a niveles superiores de educación, hasta alcanzar los niveles de especialización.

Por lo señalado anteriormente, resulta claro que la organización educativa plantea problemas y situaciones específicas, dependiendo del nivel educativo de que se trate, razón por la cual, al ser éstas entes reales y claramente diferenciados, hacen posible que la Administración Educativa posea un objeto de estudio definido y real.

\section{d. Está ontológica y epistemológicamente definida}

Como pudo verse en el apartado sobre el objeto de estudio de la Administración Educativa, el hecho de que tenga un objeto de estudio real, con su especificidad frente a cualquier otra organización posible, le confiere a la Administración Educativa definición ontológica y epistemológica. Ontológica, en cuanto hay un ente real de estudio, y epistemológica en la medida en que existen definiciones particulares del objeto de estudio, así como de los mecanismos para poder conocerlo. Por esta razón, es posible, dado el grado de desarrollo disciplinar de la Administración en general y de la Administración Educativa en particular, distinguir una organización educativa de cualquier otro tipo de organización.

\section{e. Sus teorías y métodos permiten explicar la realidad y actuar sobre ella}

Los distintos métodos y teorías empleados por los especialistas en Administración Educativa, permiten explicar la realidad de la organización educativa y actuar sobre ella, a fin de que pueda cumplir con los objetivos para los cuales fue creada. Además, estas teorías y métodos responden a las necesidades de explicar la naturaleza de los fenómenos organizacionales (dimensión ontológica), y de abordarlos de manera concreta a través de mecanismos debidamente desarrollados (dimensión metodológica), coherentes con los fundamentos teóricos y epistemológicos (cómo se puede conocer el fenómeno).

La principal característica de una disciplina científica es la coherencia interna entre los fundamentos epistemológicos y teóricos, las prescripciones metodológicas y las proyecciones de aplicación. En este sentido, se cuenta con un amplio desarrollo en la Administración Educativa.

\section{f. Dispone de un "cuerpo" de datos, hipótesis, métodos, instrumentos, entre otros, suficiente para operar, con medios para mantenerlo actualizado}

Existe un consistente acervo de datos, hipótesis, métodos e instrumentos que 
no sólo permiten la operacionalización de la disciplina, sino también una importante producción de nuevos conocimientos que actualizan, completan y mejoran los ya existentes en el campo de la Administración Educativa. La mejor evidencia de esto la constituyen los trabajos de investigación realizados por especialistas y por estudiantes de grado y posgrado.

Además, es importante considerar el aporte a nivel teórico, metodológico y técnico de las disciplinas que han contribuido a construir la teoría general sobre la organización educativa, como, por ejemplo: el Derecho, las Ciencias Políticas, la Administración Pública, la Administración Empresarial, La Teoría General de Sistemas, la Psicología, la Antropología y la Sociología, entre otras.

\section{g. La problemática que aborda se refiere a objetos que forman parte de su dominio, o a otros con los que estos objetos mantienen relaciones significativas}

La Administración educativa se ha interesado por investigar diversos aspectos adyacentes a su propio quehacer como la legislación, los actores sociales que conforman el proceso enseñanza-aprendizaje, la estructura del sistema educativo nacional, entre otros. Por esta razón, puede dar cuenta de estos en función de su propio objeto de estudio.

Como se pudo ver en el apartado anterior, la Administración Educativa, en tanto ciencia social, recibe un aporte significativo de otros campos de estudio de las ciencias sociales que, de una forma u otra, contribuyen a una mejor explicación y comprensión de la organización educativa en su especificidad.

\section{h. Cuenta con un fondo de conocimientos, cuya dimensión crece constantemente}

En la actualidad existe una amplia y diversa gama de publicaciones especializadas a nivel mundial. El volumen, dimensión y diversidad de este material crece constantemente. Por esta razón, se puede afirmar que la Administración Educativa se encuentra en una etapa de amplia producción teórica que pone en envidencia un estadio de madurez y desarrollo disciplinar. Como evidencia de ésto, se invita al lector o lectora a ingresar a Internet y verificar la cantidad de información especializada disponible en inglés y en español, así como en otros idiomas, sobre diversos aspectos propios de la disciplina. Además, existe bibliografía convencional especializada disponible en bibliotecas, compuesta por tesis, proyectos de investigación, revistas y libros producidos nacional e internacionalmente.

\section{i. Los objetivos que se persiguen hacen referencia a los objetos de su dominio: descubrimiento de leyes, formulación de teorías, diseño de métodos, entre otros}

Como se mencionó en el apartado anterior, existe investigación en un grado suficiente como para llegar a un conocimiento profundo, sistemático y articulado de la organización educativa, así como de los aspectos de gestión asociados al desempeño de la misma. En este sentido, se cuenta con teorías, métodos y técnicas específicas para ser referidos y utilizados en el campo de la Administración Educativa, orientados a la solución de problemas y al mejoramiento continuo de la práctica profesional en el campo.

\section{j. Los métodos que utiliza son escrutables y justificables}

Es un hecho que la rigurosidad en la producción de conocimiento nuevo en el campo de la Administración Educativa, permite que sus métodos sean no sólo escrutables, sino también justificables y reproducibles en situaciones similares. 


\section{La noción de paradigma de Thomas Kuhn}

Otro criterio importante para determinar si se está o no en presencia de una disciplina científica es el establecido por el epistemólogo Thomas Kuhn (1975), que tiene que ver con la noción de paradigma o matriz disciplinar. Como vimos anteriormente, para este autor los paradigmas son la forma aceptada al interior de una comunidad científica de producir los conocimientos propios de la disciplina.

Las disciplinas o matrices disciplinares deben tener los siguientes elementos:

\section{Esquema No. 2}

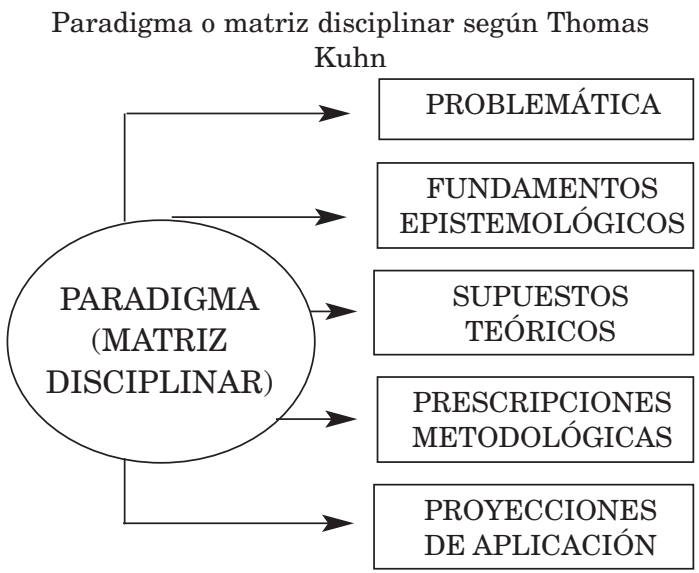

Adaptado de: Hernández, 1998.

Como puede observarse, la Administración Educativa también cumple con los requisitos estipulados por Kuhn para que exista una disciplina científica. Así:

1. Cuenta con una problemática: Estudio y análisis de problemas (enigmas) teóricos y experimentales que define un paradigma, para los cuales hay que aportar soluciones (ejemplares). Dicha solución a los problemas o problemáticas que define la matriz discliplinar aporta y amplía sus coordenadas teórico-explicativas.
2. Cuenta con fundamentos epistemológicos: Son supuestos más generales a los anteriores, con los cuales se compromete la comunidad adepta al paradigma. En esencia tienen que ver con aspectos de tipo metateórico, vinculados con concepciones epistemológicas (definición de objeto de estudio, concepto de realidad, lógica heurística, entre otros.)

3. Tiene fundamentos teóricos: Los programas de investigación están asociados a aquellas hipótesis o sustentos teóricos que comparten los especialistas y que son la base fundamental de la matriz disciplinar.

4. Tiene prescripciones metodológicas: Los métodos, técnicas y reglas aceptadas por la comunidad de profesionales, que se utilizan en el trabajo de construcción y refinamiento (investigación) del paradigma.

5. Tiene proyecciones de aplicación: Son las técnicas o tecnología necesarias para la aplicación de los supuestos teóricos que propone el paradigma a situaciones reales. (Cfr.: Hernández, 1998).

Con base en lo expuesto hasta aquí, resulta claro que la Administración Educativa es una disciplina compleja que involucra lo educativo, lo administrativo y a otras disciplinas, sin perder por ello su especificidad (nivel ontológico). Además, se puede afirmar que cumple con los indicadores establecidos por Mario Bunge (Gómez, 1996) y por Thomas Kuhn (1975), para determinar si se está o no en presencia de una disciplina científica. En este sentido, se considera que no sólo cumple con estos requerimientos, sino que la Administración Educativa se encuentra en una etapa de madurez disciplinar que la conduce, sin lugar a dudas, a una creciente 
producción de conocimientos nuevos e innovadores, capaces de responder a las nuevas y complejas demandas sociales de educación, así como sobre la cantidad y la calidad de la oferta educativa requerida en la época actual.

Para resumir, como puede verse en el esquema No. 3, como componentes de la organización educativa podemos reseñar los siguientes:

Esquema No. 3

Componentes de la organización educativa

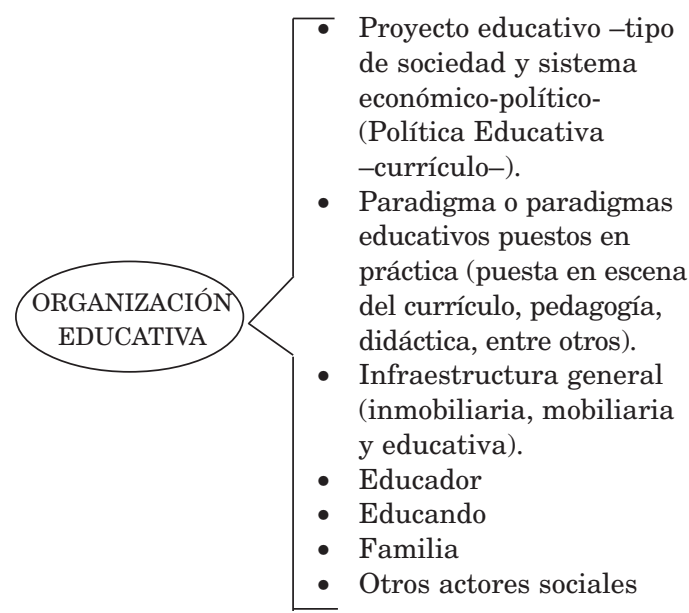

Con base en el esquema anterior se pueden distinguir los elementos que componen la organización educativa y, por ende, los aspectos a considerar en la configuración teórico-metodológica y práctica de la disciplina.

La Administración Educativa se encarga, por lo tanto, de administrar el currículum, con base en determinado paradigma educativo. Esto implica que la disciplina debe encontrar el paradigma administrativo coherente con los objetivos curriculares del paradigma educativo al que se adscriba.

Con base en lo expuesto hasta aquí, podemos decir que la Administración Educativa forma parte del conjunto de disciplinas que componen las Ciencias de la Educación, ya que, como pudo observarse, posee su propio objeto de estudio, su propia teoría, y sus métodos y técnicas de investigación, derivados de la plasticidad paradigmática con que cuenta la teoría educativa, como lo muestra el esquema No. 4:

$$
\text { Esquema No. } 4
$$

Disciplinas que componen las Ciencias de la Educación

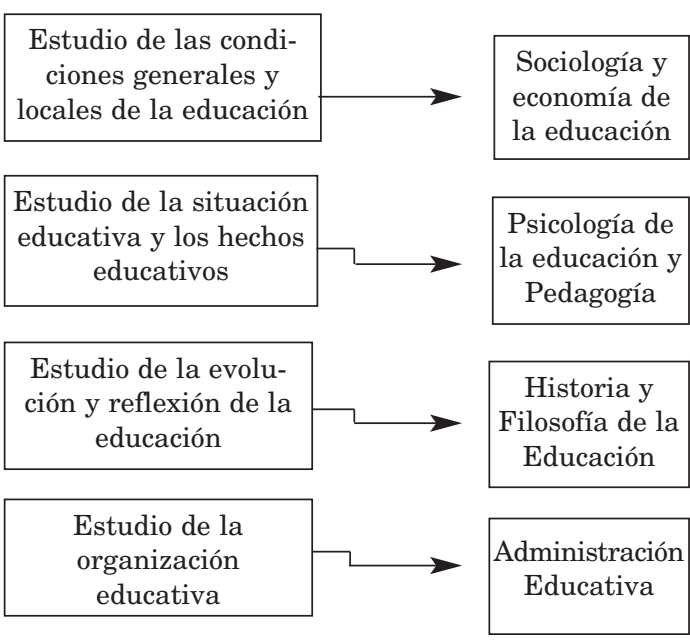

Adaptado de: Hernández, 1998.

La Administración Educativa no sólo ha tenido un importante auge en la última década del siglo pasado, sino que se perfila como una disciplina de gran interés investigativo y crítico en la década presente, debido a los retos que enfrenta la educación en la actualidad. Es indispensable elevar la calidad de la educación, razón por la cual elevar la capacidad de gestión de la organización educativa se vuelve uno de los principales problemas a resolver por parte de los teóricos e investigadores de este campo.

Sin duda alguna, en los próximos años veremos un crecimiento significativo de la investigación, la producción teórica y la aplicación de esta disciplina, que contribuirá a hacer los cambios que requiere la educación para enfrentar los retos de un mundo globalizado, en el que se espera alcanzar un desarrollo sostenible con equidad y justicia social. 


\section{Referencias bibliográficas}

Bunge, Mario. La investigación científica. México: Ariel, 1992.

Chiavenato, Idalberto. Introducción a la teoría administrativa. Colombia: MacGraw-Hill, 1995.

Flores Ochoa, Rafael. Evaluación pedagógica y cognición. Colombia: MacGrwHill, 2000.
Gómez Dacal, Gonzalo. Curso de organización escolar y general. España: Editorial Escuela Española, 1996.

Hernández Rojas, Gerardo. Paradigmas en Psicología de la Educación. México: Paidós, 1998.

Kuhn, Thomas S. La estructura de las revoluciones científicas. Madrid: Fondo de Cultura Económica, 1975.

Flora Salas Madriz Profesora de la Escuela de Administración Educativa de la Universidad de Costa Rica 